\title{
INTUITIONISTIC COMPLETENESS \\ FOR FIRST ORDER CLASSICAL LOGIC
}

\author{
STEFANO BERARDI
}

\begin{abstract}
In the past sixty years or so, a real forest of intuitionistic models for classical theories has grown. In this paper we will compare intuitionistic models of first order classical theories according to relevant issues, like completeness (w.r.t. first order classical provability), consistency, and relationship between a connective and its interpretation in a model. We briefly consider also intuitionistic models for classical $\omega$-logic.

All results included here, but a part of the proposition (a) below, are new. This work is, ideally, a continuation of a paper by McCarty, who considered intuitionistic completeness mostly for first order intuitionistic logic.
\end{abstract}

§1. Introduction. Here is a list of propositions we will prove. Let $T$ be any classical theory.

PROPOSITION (a). If $T$ is recursive, there are quite natural classes of models of $T$, like Tarski models, which are intuitionistically complete only in the case $T$ is decidable (thus, not very often). ${ }^{1}$

Proposition (b). The main result of the paper is: if we want a class of model of $T$ to be complete, and each model of the class to be consistent, then, in general, we have to interpret classical $\neg, \rightarrow$ by connectives intuitionistically strictly stronger than $\neg$, $\rightarrow ;$ and $\vee, \exists$, by connectives intuitionistically strictly weaker than $\vee, \exists$.

Many classes of models of this kind are known, all more or less inspired by KripkeBeth models (for which we refer to [11]).

Proposition (c). The only other way to have completeness, if we still want the interpretation of classical $\neg$ to be intuitionistically comparable with (metatheoretical) $\neg$, is to drop the consistency requirement on models. In this case interpret classical $\neg$ by a connective intuitionistically strictly weaker than $\neg$.

Only one class of models of this kind is known, defined by Krivine in [5].

Apparently (but we will discuss the matter again), the classes of models closest to the original meaning of the classical theory they are interpreting are those where each model is consistent. Proposition (b) describes some features they must have.

Our results apply as well to Tait's classical $\omega$-logic [10] and to single models (i.e., to classes including only one model). Since $\omega$-provability is classically equivalent

Received October 16, 1996; revised July 23, 1997; revised September 30, 1997.

${ }^{1}$ Added in proof. Some of the intuitionistic incompleteness results are proved, in a different way, in [6]; see Section 3. 
to classical truth, they in fact apply to any intuitionistically complete single model of the notion of classical truth. There is, up to equivalence, a unique model of this kind, falling in case (b) (see the end of section 4).

This is the plan of the paper. In section 2 we introduce the notion of model, of intuitionistic model theory, and the properties of models we will speak of. In section 3 we derive some preliminary remarks about completeness. In section 4 we derive Propositions (a), (b), (c) above, and we briefly discuss them. All proofs in this paper are intuitionistic.

§2. Intuitionistic model theory. We suppose fixed a language $\mathbf{L}$ for first order classical logic CL. We suppose having in CL only closed formulas. The connectives $c$ of $\mathbf{L}$ are:

$$
c=\perp, \neg, \wedge, \vee, \rightarrow, \exists, \forall
$$

A "theory" $T$ will be any set of formulas of $\mathbf{L} ; T$ is recursive if it is recursive as set. By $P A$ we will denote the (recursive) set of axioms of Peano Arithmetic. We consider two notions of (classical) proofs for $T$ : first order proof (under any formalism), and infinitary proofs a la Tait (obtained by adding the $\omega$-rule: derive $\forall x . A[x]$ from $A[t]$ for all term $t$ of $\mathbf{L}$ ). In both cases, we denote " $p$ is a classical proof of $A$ in $T$ " by " $p, T \vdash_{c} A$ ", and " $(\exists p)\left(p, T \vdash_{c} A\right)$ " by " $T \vdash_{c} A$ ". When we want to precise if we are speaking about first order or infinitary deduction, we will write respectively $T \vdash_{1} A$ and $T \vdash_{\omega} A$. We will drop the index $T$ when no confusion arise.

DEFINITION 2.1 (Interpretation of $\mathbf{L}$ ). Let $M$ be any predicate on $\mathbf{L} ; M$ may be considered as a map: $\mathbf{L} \rightarrow$ Propositions $\}$. We will denote $M(A)$ by $M \models A$, to be read " $A$ is true in $M$ "; we will say that $M$ is an interpretation for $\mathbf{L}$.

If $C$ is any class of interpretations, we will denote " $\forall M \in C . M \models A$ " by " $C \models A$ ", and we will read it " $A$ is valid in $C$ ".

We only model the satisfiability relation of $M$, in other words, the set of formulas satisfied by $M$. We did not define explicitly the inhabitants of a model. This for sake of generality: we want our result be valid for Tarski models, Kripke models, topological models, and so on; it would be hard to provide a definition of inhabitant encompassing them all. ${ }^{2}$

In fact, since $M$ is just a predicate, i.e., a set of formulas, then $M$ is just a generic theory over the language $\mathbf{L}$, used to interpret another one. $M$ could be the set of formulas true in some structure, but $M$ could also be any translation $\psi: \mathbf{L} \rightarrow$ \{Propositions\}, say $\psi=$ Gödel $\neg$-translation. In this case $(M \models A) \leftrightarrow \psi(A)$, that is, $M \vDash A$ means that $\psi(A)$ holds.

Argument of this paper is what we can and what we cannot derive in the intuitionistic theory of the interpretations of $\mathbf{L}$, theory we will call, from now on, "Intuitionistic Model Theory". In order to prove that something is not derivable in Intuitionistic Model Theory, we have to choose a formalism for this latter; we chose Second Order Intuitionistic Arithmetic $H^{2} A$. For instance, we will suppose a class $C$ of models formalized by a second order formula with free variable $M$. Many

\footnotetext{
${ }^{2}$ If we want, we might identify the inhabitants of $M$ with the terms of $\mathbf{L}$. This convention does not forbid us to consider interpretations including elements not denoted by terms of $\mathrm{L}$ : we have just to consider them terms of some $\mathbf{L}^{\prime}$ including $\mathbf{L}$.
} 
other choices are possible (Martin-Löf Type Theory, Intuitionistic Set Theory, and so on), and there is no special purpose in the one we did. All proofs of this paper rely on quite general considerations, and would still carry on for a different formalism.

Thus, when we will assert that some result about interpretations holds intuitionistically, or fails intuitionistically, or holds classically, or it is consistent with intuitionism, strictly speaking we will mean that such result is, respectively: provable in $H^{2} A$, not provable in $H^{2} A$, provable in $H^{2} A+$ Excluded Middle, consistent with $H^{2} A$. When we will say that a statement or predicate is intuitionistically strictly stronger (weaker) than another, we will mean that one implication is provable in $H^{2} A$, while the opposite one is not. Yet, the reader may intend the word "intuitionism" in its broader sense without being wrong.

We introduce now the properties of interpretations we will speak about in this paper.

DEFINITION 2.2 .

1. We say that $M$ is a sound interpretation of $T$ (and $\vdash_{c}$ ) if and only if for all $A \in \mathbf{L}:\left(T \vdash_{c} A\right) \Longrightarrow(M \models A)$.

2. We say that $M$ faithfully interprets an intuitionistic connective $c$ if and only if " $M$ interprets the intuitionistic $c$ by the classical $c$ ". This is to say: $M$ faithfully interprets $c$ if for all $A, B \in \mathbf{L}$ we have, according to what connective $c$ is:

$$
\begin{aligned}
\perp & \Longleftrightarrow M \models \perp \\
\neg(M \models A) & \Longleftrightarrow M \vDash \neg A \\
(M \models A) \wedge(M \models B) & \Longleftrightarrow M \models A \wedge B \\
(M \models A) \vee(M \models B) & \Longleftrightarrow M \models A \vee B \\
((M \models A) \Longrightarrow(M \models B)) & \Longleftrightarrow M \vDash A \rightarrow B \\
\exists t:(M \models A[t]) & \Longleftrightarrow M \models \exists x \cdot A[x] \\
\forall t:(M \models A[t]) & \Longleftrightarrow M \models \forall x \cdot A[x]
\end{aligned}
$$

Note that the connectives on the left-hand-side have to be understood intuitionistically (they are connectives in Intuitionistic Model Theory), and those in the right-hand-side classically (they are connectives in $\mathbf{L}$ ) $\exists t, \forall t$ range over closed terms of $L$.

3. We say that $M$ is a sound (complete) interpretation of a connective $c$ iff $M$ interprets the intuitionistic $c$ by an operation on propositions of $\mathbf{L}$ weaker (stronger) than $c$. This is to say: in the table above, the corresponding left-to-right (right-toleft) implication holds (intuitionistically).

4. We say that $M$ is a model of $T$ (and $\vdash_{c}$ ) iff $M$ is a sound interpretation of $T$, $\vdash_{c}$ which is complete for $\rightarrow$, i.e., which satisfies modus ponens:

$$
M \models A \rightarrow B, \quad M \models A \Longrightarrow M \models B .
$$

5. We say that $M$ is consistent iff $\neg(M \models \perp)$, that is, if and only if $M$ is complete for $\perp$.

6. Let $C$ be any class of models of $T$. We say that $M$ is complete for $T$ (and $\vdash_{C}$ ) if and only if for all $A \in \mathbf{L}$ we have:

$$
C \models A \Longrightarrow T \vdash_{c} A .
$$


A single model $M$ is complete if and only if the class consisting of $M$ alone is. A class $C$ is classically complete if and only if completeness may be proved classically.

In all models we may prove, using soundness and modus ponens, that $\perp, \vee, \exists$ are sound, $\forall, \rightarrow$ are complete, while $\wedge$ is faithful, that is,

$$
(M \vDash A) \wedge(M \vDash B) \Longleftrightarrow(M \vDash A \wedge B) \text {. }
$$

In any model completeness for $\perp$ (or "consistency") and for $\neg$ are equivalent. (Proof. Out of $M \models \neg \perp$ and completeness for $\neg$ we may prove $\neg(M \models \perp$ ). Conversely, if we assume $M \models \neg A$, out of soundness we have $M \models(\neg A \rightarrow A \rightarrow \perp)$, by modus ponens $M \models(A \rightarrow \perp)$, by modus ponens again $(M \models A) \rightarrow(M \models \perp)$, and by consistency, $\neg(M \models A)$.)

The classes of models and single models considered in literature are, often, intuitionistically sound, classically complete, but not intuitionistically complete. This is the case of Tarski models for first order theories (see section 4). This is also the case of Gödel $\neg \neg$-interpretation and Dialectica Interpretation for $\omega$-logic for $P A$ (again, see section 4).

In the rest of the paper we will prove that, in order to have intuitionistic completeness, we have to interpret negation in a non-faithful way. We will then classify the intuitionistically complete classes of models according if models are sound or complete for negation. In the first case intuitionistic negation is stronger than negation in the model, in the second one is weaker. All intuitionistically complete interpretations we know about, but one, will fall in the second case.

§3. Some preliminary results. In this section we will restate results from literature in term of completeness for connectives. ${ }^{3}$ Out of the Lemmas in this section we will derive the propositions listed in the introduction.

Lemma 3.1 (Main Lemma). Let $\vdash_{c}$ be $\vdash_{1}$ or $\vdash_{\omega}$. In Intuitionistic Model Theory, for no class $C$ of models of $T=P A$ we may prove both that $C$ is complete for $T \vdash_{c}$, and that any $M \in C$ interprets $\neg$ faithfully.

Proof. Suppose such a class $C$ exists. Take any $A \in \mathbf{L}$ and any $M \in C$. By soundness, $M \models \neg \neg A \rightarrow A$. Out of it, by modus ponens for $M$, and

$$
\neg(M \models A) \Longleftrightarrow M \models \neg A,
$$

we derive $\neg \neg(M \models A) \Longrightarrow M \models A$. From this latter, we intuitionistically have

$$
\neg \neg \forall M \in C .(M \models A) \Longrightarrow \forall M \in C .(M \models A)
$$

for all $M \in C$, that, is, $\neg \neg C \models A \Longrightarrow C \vDash A$. Now by completeness we have $C \models A \Longleftrightarrow\left(\vdash_{c} A\right)$, and we conclude $\neg \neg\left(\vdash_{c} A\right) \Longrightarrow\left(\vdash_{c} A\right)$. Therefore

$$
\neg \neg\left(\vdash_{c} A\right) \Longleftrightarrow\left(\vdash_{c} A\right)
$$

holds in intuitionism.

This is absurd, no matter if $\vdash_{c}$ is $\vdash_{1}$ or $\vdash_{\omega}$. In order to see it, take $A=A[x, y]=$ $\exists z . T[x, y, z]$, where $T[x, y, z]$ is the Peano formula representing the total recursive predicate: "the $x$-th partial recursive map, applied to $y$, converges in $z$ step". Since

\footnotetext{
${ }^{3}$ Lemmas similar to 3.1 and 3.3 are, with different statements and proof, in McCarty [6], p. 560, and [7], 13th page. A kind of weaker form of Lemma 3.2 is in [6], p. 562.
} 
$\vdash_{c}$ is either first order or infinitary deduction from $P A$, and $A$ is simply existential, we have $\left(\vdash_{c} A\right) \leftrightarrow A$ for all $x, y$. (Proof: left-to-right: by using Novikoff-Coquand Game interpretation, or in many other ways; right-to-left: easy.) Out of it and $\neg \neg\left(\vdash_{c} A\right) \Longleftrightarrow\left(\vdash_{c} A\right)$ we prove $\neg \neg A \leftrightarrow A$. Thus, we may realize this statement in the Typed Realization Model for $H^{2} A$, the theory we are using as Intuitionistic Model Theory (see [8]). In this Model holds the principle of Independence from the Hypothesis:

$$
(\neg B \rightarrow \exists z . C[z]) \rightarrow(\exists z . \neg B \rightarrow C[z])
$$

(for $z$ not free in $B$; see [8], chapter 1 for a proof). If we take now $B=\neg A, C[z]=$ $T[x, y, z]$, in the same Model from $\neg \neg A \rightarrow A$ we obtain $\exists z .(\neg \neg A \rightarrow T[x, y, z])$ and, since $T[x, y, z] \rightarrow \neg \neg A$ intuitionistically, also $\exists z$. $(\neg \neg A \leftrightarrow T[x, y, z])$ This means that there is a total recursive map $x, y \mapsto z(x, y)$, and a realizer of: $\neg \neg A \leftrightarrow$ $T[x, y, z(x, y)]$ and by $\neg \neg A \leftrightarrow A$, also of $A[x, y] \leftrightarrow T[x, y, z(x, y)]$. We may decide whether we may realize $T$, therefore we may decide whether we may realize $A$. But $A$ is realizable if and only if it holds, so $A$ is decidable. This is absurd, because the meaning of $A$ is: "the $x$-th partial recursive map, applied to $y$, converges". ${ }^{4}-1$

The Main Lemma says that if we want to have both completeness, and the interpretation of $\neg$ comparable with $\neg$ (intuitionistically), then, in general, we must interpret $\neg$ by a connective either strictly weaker or strictly stronger than $\neg$ (again, intuitionistically).

We will draw some more consequences out of the Main Lemma in Section 4. Before we will precise, in the case $T$ is recursive and $t_{c}$ is first order deduction, for what $T$ we intuitionistically have both completeness and a faithful interpretation of negation.

Lemma 3.2. Let $T$ be any recursive theory. In Intuitionistic Model Theory, if we may prove that there is a complete class $C$ of models of $T$ and $\vdash_{1}$, which interpret negation faithfully, then $T$ is a decidable first order theory.

Proof. As we did in Lemma 3.1, we may derive $\neg \neg\left(\vdash_{1} A\right) \leftrightarrow\left(\vdash_{1} A\right)$ for any $A$, and then realize this statement in the Typed Realization Model. By definition, $\left(\vdash_{1} A\right)=\exists p \cdot\left(p \vdash_{1} A\right)$. If we use again the principle of Independence from the Hypothesis:

$$
(\neg B \rightarrow \exists z . C[z]) \rightarrow(\exists z . \neg B \rightarrow C[z])
$$

(for $z$ not free in $B$ ), we may realize also the statement: $\exists p .\left(\neg \neg\left(\vdash_{1} A\right) \rightarrow\left(p \vdash_{1} A\right)\right.$ ) for all $A$ in $\mathbf{L}$. By definition of Realization, this means that there is some recursive map $A \mapsto p(A)$ such that for all $A$ in $\mathbf{L}$ we have in the Realization Model:

$$
\neg \neg\left(\vdash_{1} A\right) \rightarrow\left(p(A) \vdash_{1} A\right) .
$$

Since $\left(p(A) \vdash_{1} A\right) \rightarrow\left(\vdash_{1} A\right)$ by logic, and $\left(\vdash_{1} A\right) \rightarrow \neg \neg\left(\vdash_{1} A\right)$, we also realize: $\left(\vdash_{1} A\right) \leftrightarrow\left(p(A) \vdash_{1} A\right)$ for all $A$. By intuitionistic logic, there is a realizer of $\left(p(A) \vdash_{1} A\right) \vee \neg\left(p(A) \vdash_{1} A\right)$ (being a first-order proof of a given $A$ is decidable

\footnotetext{
${ }^{4}$ It is worth noticing that an alternative proof of Lemma 3.1 may be obtained by adapting Gödel's argument for intutionistic underivability of completeness for first order intuitionistic logic. Out of $\neg \neg\left(\vdash_{c} A\right) \rightarrow\left(\vdash_{c} A\right)$ we may intuitionistically derive Markov's principle, and from the intuitionistic underivability of this latter the intuitionistic underivability of the former.
} 
because $T$ is a recursive set of axioms). Thus, we may realize $\left(\vdash_{1} A\right) \vee \neg\left(\vdash_{1} A\right)$, that is, we may decide realizability of $\left(\vdash_{1} A\right)$. Since we may realize $\left(\vdash_{1} A\right)$ if and only if $\left(\vdash_{1} A\right)$ holds, eventually we conclude that we may decide $\left(\vdash_{1} A\right)$.

We only quote a stronger version of Lemma 3.2: "In Intuitionistic Model Theory, we may prove that there is a complete class $C$ of models of $T$ and $\vdash_{1}$, which interpret negation faithfully, if and only if we may prove that $T$ is a decidable first order theory". We conclude Section 3 by a reformulation of a Kreisel result, included in McCarty paper [7] (13th page):

LEMMA 3.3. Let $\vdash_{c}$ be $\vdash_{1}$ or $\vdash_{\omega}, T=P A$ and $M$ a generic consistent model of $T$ and $\vdash_{c}$ (i.e., such that $\left.\neg(M \models \perp)\right)$. Then it is consistent with intuitionism to assume that $M$ is:

1. not complete for $\vee$;

2. not complete for $\exists$.

Proof. We will prove that if $T=P A$ then in the Typed Realization Model of $H^{2} A$, our Intuitionistic Model Theory, both 1, 2 hold. We will conclude that these statements are consistent with $H^{2} A$.

1. Out of soundness we have $(M \models A \vee \neg A)$. By completeness of $M$ for $\vee$, and consistency of $M$, we would get, intuitionistically, $(M \models A) \vee \neg(M \models A)$. Thus, this statement is realizable, and so $M=\{A \mid M \models A$ is realizable $\}$ is a decidable set, including the first order theorems of $T=P A$, closed by modus ponens, and not including $\perp$ (by consistency of $M$ ). This contradicts Gödel's Undecidability Theorem.

2. Out of soundness we have

$$
M \vDash \exists x .(x<2) \wedge(x=0 \rightarrow A) \wedge(x=1 \rightarrow \neg A) .
$$

By completeness of $M$ for $\exists$, modus ponens, and consistency we would get for some $x$ :

$$
(M \models x<2), \quad(M \models x=0) \rightarrow(M \vDash A), \quad(M \models x=1) \rightarrow \neg(M \models A)
$$

Since $(M \models A t) \leftrightarrow A t$ for any $A t$ closed atomic formula, and any consistent model $M$ of $P A$, we would have again, intuitionistically, $(M \models A) \vee \neg(M \models A)$ for all $A \in \mathbf{L}$.

§4. Conclusions. From Lemma 3.1 we derive now Proposition (a) of the introduction. In fact, an important example of classes of models of $T$ interpreting $\neg$ faithfully (and therefore, in general, not intuitionistically complete) are Tarski classes models, which we will now introduce from an intuitionistic viewpoint.

Tarski defines his models starting from a set $S$ of classic connectives, from which all others connectives in $\mathbf{C L}$ are definable. We call such sets of connectives complete. Examples are

$$
S_{0}=\{\exists, \vee, \neg\}, \quad S_{1}=\{\forall, \rightarrow, \perp\}, \quad S_{2}=\{\forall, \wedge, \neg\}, \quad S_{3}=\{\forall, \rightarrow, \neg\}, \quad \ldots
$$

Let $\phi_{S}: \mathbf{L} \rightarrow$ formulas containing only connectives in $\left.S\right\}$ be the map defined replacing each connective $c \notin S$ with the connectives of $S$ defining $c$ (in CL). For instance, if $S=S_{2}$, then

$$
\phi_{S}(A \vee B)=\neg\left(\neg \phi_{S}(A) \wedge \neg \phi_{S}(B)\right) .
$$


Definition 4.1. Let $T$ be any theory. We will say that $M$ is a Tarski model for $T$ and $S$ if and only if:

1. $T \subseteq M$, and $M$ faithfully interprets each connective in $S$.

2. For all $A \in \mathbf{L}$, all connectives not in $S$ are interpreted as abbreviations of the connectives in $S$ defining it. We may express this by saying:

$$
M \vDash A \Longleftrightarrow M \models \phi_{S}(A) .
$$

3. $M$ satisfies some formulation of Excluded Middle expressed using only the connectives in $S$. For instance, if $S=S_{1}, S_{3}$ we require $M \models$ PierceLaw, while if $S=S_{2}$ we ask

$$
\neg \neg(M \models A t) \rightarrow(M \models A t)
$$

for all atomic formulas $A t$ of $\mathbf{L}$.

In the definition of Tarski models we may find, say, in [9], chapter 2, conditions 2, 3 are missing. Yet, 2 is implicitly introduced when it is said that the connectives not in $S$ may be introduced as abbreviations; and 3 is implicitly present because the Model Theory considered by Tarski is classical.

Tarski models are models also in our sense. Any complete set $S$ of classical connectives either contains $\neg$ or both $\rightarrow$ and $\perp .{ }^{5}$ In both cases all Tarski models for $S$ faithfully interprets $\neg$ (Proof. In the first case, this holds by definition of Tarski model, in the second one, by $M \models((A \rightarrow \perp) \leftrightarrow \neg A)$, modus ponens, and faithfulness for $\rightarrow, \neg$.) Thus, by Lemma3.1 for no $S$ the class of Tarski models for $T$ and $S$ may intuitionistically be proved complete. From this and Lemma 3.2 we derive Proposition (a) of the Introduction.

We will now draw some more consequences out of Lemma 3.1. We already said that, in general, if we want intuitionistic completeness for a class $C$ of models of $T$ we have to interpret intuitionistic negation by a classical negation which, if it is comparable at all with the original one, it is intuitionistically either strictly weaker or strictly stronger. In first case we have soundness but not completeness, in the second one completeness but not soundness for $\neg$.

In the second case, we have consistency for all $M$ in $C$ (implied by completeness for $\neg$ ). Soundness for $\rightarrow$ (intuitionistically) fails (it would imply soundness for $\neg$ ). Thus $\rightarrow$ is interpreted in any $M \in C$ by a connective (intuitionistically) strictly stronger than $\rightarrow$. Also completeness for $\vee, \exists$ in general fail (intuitionistically), by consistency of all $M$ and Lemma 3.3.

What we just remarked is the content of Proposition (b) of Introduction. Almost all intuitionistically complete class of models of $T, \vdash_{1}$ which are known, like Beth models in Trölstra-Van Dalen book [11], fall within this second case. In other words, they satisfy

$$
(M \models \neg A) \rightarrow \neg(M \models A)
$$

but not the reverse. In general, in such interpretations to affirm $\neg A$ is stronger (more informative) then just to negate $A$.

\footnotetext{
${ }^{5}$ Suppose $\neg \notin S$, and either $\rightarrow \notin S$ or $\perp \notin S$. Then in the case $\neg, \rightarrow \notin S$ the set $S$ cannot define $\neg$; in the case $\neg, \perp \notin S$, the set $S$ cannot define $\perp$.
} 
Only one class of models falling within the first case is known. It was introduced by Krivine in [5], and in our terminology it is defined by:

$$
K=\{M \mid M \text { sound interpretation of } T \text { faithfully intepreting } \forall, \rightarrow\} .
$$

$K$ is a class of models in our sense, and Krivine proved it complete by a subtle constructivisation of Gödel's completeness proof. For any $A$, he defined a model $M$ such that $M \models \neg A$, and $(M \models \perp) \leftrightarrow\left(\vdash_{1} A\right)$. Thus, if $A$ is valid, then $M \models A$, and out of $M \models A, M \models \neg A$ he derives $M \models \perp$, that is, $\left(\vdash_{1} A\right)$. In Krivine's models we have:

$$
\begin{aligned}
\neg(M \models A) & \Longleftrightarrow((M \models A) \Longrightarrow \perp) \Longrightarrow((M \models A) \Longrightarrow(M \models \perp)) \\
& \Longleftrightarrow(M \models A \rightarrow \perp) \\
& \Longleftrightarrow(M \models \neg A),
\end{aligned}
$$

but not the reverse. In this model, to negate $A$ is stronger than to affirm $\neg A$. Consistency is false: we may have $M \models \perp$, and in this case, $M \models A$ for all $A \in \mathbf{L}$.

Discussion. Completeness seems a relevant issue, in order to have an intuitionistic interpretation of $A$ close to the original classical meaning of $A$. So we must choose if we prefer the interpretations with an intuitionistic negation weaker or stronger than the negation in $\mathbf{L}$ (than classical negation).

It seems to the author that if we are interested to information carried by a connective, we should consider classical negation stronger than intuitionistic negation. Indeed, intuitionistic negation is not informative, while classical negation may produce statements containing information. For instance, if we negate $\forall x \cdot p(x)$, with $p$ decidable, we obtain a formula equivalent to $\exists x . \neg p(x)$, whose proofs contain, according to Proof Theory, the value of $x$ such that $p(x)$. Thus, if we use information as a meter, we should choose completeness for $\neg$ in our models, and drop out soundness. Still, information is not the only parameter of judgment: also being able to constructivize the main proofs of classical Model Theory is a very relevant issue. And if we use the class $K$ of Krivine's models, we may turn Gödel Completeness proof into a constructive argument.

We will now derive some corollaries concerning classical $\omega$-provability $\vdash_{\omega}$ (equivalently, classical truth). Suppose be given any intuitionistically sound and complete single model for $\vdash_{\omega}$ and $P A$. This model, call it $M$, satisfies: $(M \models A) \Longleftrightarrow\left(\vdash_{\omega} A\right)$ and so it is unique up to intuitionistic equivalence, and it is also consistent. Only two non-trivial descriptions of $M$ are known: Novikoff-Coquand game model [2], proved intuitionistically complete by $\mathbf{H}$. Herbelin in [3], and Kreisel no-counterexample interpretation [4], [9], proved intuitionistically complete in [1]. By uniqueness of $M$, these two interpretations are intuitionistically equivalent. Intuitionistic completeness for $\vdash_{\omega}$ is not easy thing to get: for instance, Gödel $\neg \neg$-interpretation, and Gödel functional interpretation, described in [9], are not intuitionistically complete for $\vdash_{\omega}$. The first claim follows from Lemma 3.1, since $\neg \neg$-interpretation is trivially faithful for $\neg$. For a proof of the second claim, we refer to [4], note of p. 261, or to the appendix of [1]. 
By completeness and consistency of $M$, by Lemmas 3.1, 3.3, what we know in general about models, and that fact that $\vdash_{\omega}$ satisfies $\omega$-rule, we may derive the following information about $M$.

1. $M$ faithfully interprets $\forall, \wedge, \perp$;

2. $M$ interprets classical $\vee, \exists$ by connectives intuitionistically strictly weaker than $\vee, \exists ;$

3. $M$ interprets classical $\neg, \rightarrow$ by connectives intuitionistically strictly stronger than $\neg, \rightarrow$.

This result is exactly what we should have expected. In order to convince ourself of this point, we have only to compare the notion of classical truth for $\mathbf{L}$, modeled in $M$, with the notion of intuitionistic truth.

1. The informal description of $\forall, \wedge, \perp$ in intuitionistic and classical logic are much the same. This explain (1).

2. A disjunction or an existential has a stronger meaning in intuitionism than in classical logic. This explain (2).

3. The intuitionistic negation of any $A$ in $M$ means not having an evidence for $M \models A$, while the classical negation of the same $A$ in $M$ means having an evidence for $M \models \neg A$. This latter statement is more informative, and thus stronger, from an intuitionistic viewpoint. This explain the first half of (3). The second half of (3) comes as consequence, since negation is as particular case of implication.

Acknowledgments. We thank Simona Ronchi for stimulating the writing out of this short note, Jan Smith, Piergiorgio Odifreddi and David McCarty for helpful discussions on the topic.

\section{REFERENCES}

[1] S. Baratella and S. Berard, Yet another constructivization of classical logic, Proceedings of 1995 Venice congress "Twenty-five years of constructive type theory".

[2] T. CoQuand, A semantic of evidence for classical arithmetic, this Journal, vol. 60 (1995), no. 1.

[3] H. Herbelin, Sequents qu'on calcule, Ph.D. thesis, University of Paris VII, January 1995.

[4] G. KREISEL, On the interpretation of non-finitist proofs, this JourNal, vol. 16 (1951), p. 261.

[5] J.-L. KRIVINE, Une preuve formelle et intuitionniste du theoreme de completude de la logique classique, Bulletin of Symbolic Logic, to appear, available by $\mathrm{ftp}$ from boole.logique.jussiue.fr in pub/distrib/krivine.

[6] MCCARTY, Undecidability and intuitionistic incompleteness, Journal of Philosophical Logic, vol. 25 (1996), pp. 559-565. 1997.

[7] - Completeness for intuitionistic logic, to appear in P. Odifreddi's book about G. Kreisel,

[8] Ch. Pauln-Mohring, Extraction de programmes dans le calcul des constructions, These de doctorat, Universite de Paris VII, 1989.

[9] J. R. SHOENFIELD, Mathematical Logic, ch. 8, Number theory, Addison-Wesley, London, 1967.

[10] W. W. TAIT, Normal derivability in classical logic: The syntax and semantic of infinitary languages, Lecture Notes in Mathematics, vol. 72, Springer-Verlag.

[11] TrÖLstra and VAN Dalen, Constructivism in Mathematics, ch. 13, Semantic completeness, North-Holland.

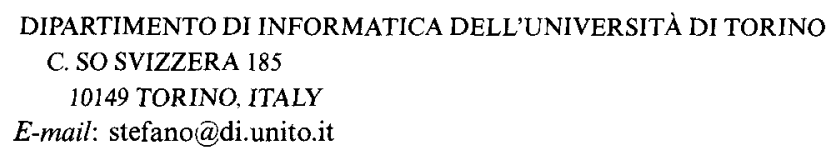

